\title{
Characterization of Interconnects Resulting from Capillary Die-to-Substrate Self-Assembly
}

\author{
Massimo Mastrangeli ${ }^{1,2}$, Wouter Ruythooren ${ }^{1}$, Chris Van Hoof ${ }^{1}$ and Jean-Pierre Celis ${ }^{2}$ \\ ${ }^{1}$ Interuniversity MicroElectronic Center (IMEC) \\ Kapeldreef 75, 3001 Leuven (Belgium) \\ ${ }^{2}$ Katholieke Universiteit Leuven, Dept. MTM \\ Kasteelpark Arenberg 44, 3001 Leuven (Belgium) \\ Email: mastran@imec.be Phone:+32-1628-8672 Fax:+32-1628-1576
}

\begin{abstract}
Capillary fluidic self-assembly (SA) intrinsically features massively-parallel, contactless die handling and allows for high-precision die placement. It may thus boost die-to-substrate assembly throughput and scalability. Here we characterize for the first time indium interconnects established between dummy dies and substrates as integral part of a capillary SA process. We present a simple way to keep the solder surface free of oxide during assembly, and we show that $\mathrm{In} / \mathrm{Au}$ wetting and bonding is not prevented by hydrocarbons and selfassembled monolayers locally present during processing. Resulting solder joints are characterized by mechanical shear tests, SEM and SAM. We assess the electrical functionality of interconnects with a simple test structure. Finally, we discuss the effects of an external load applied on dies during bonding. Our results open interesting perspectives for adopting capillary SA for die integration over non-planar substrates.
\end{abstract}

\section{Introduction}

Assembly of thin dies onto planar target substrates is critical for packaging and 3D integration of electronic systems [1]. Currently, serial techniques like pick-andplace and flip-chip perform this task satisfactorily for consumer electronic applications. Anyway, they face a trade-off between assembly throughput and registration accuracy of dies. Moreover, such techniques prove impractical in handling sub-millimetric components [2].

Fluidic self-assembly (SA) techniques may conversely outperform established approaches for dense integration of large quantities of tiny components. Particularly, in capillary fluidic SA massively-parallel and contactless die handling, which are intrinsic to fluidic SA, combine with highly-accurate die positioning [3]. The underlying concept is depicted in Fig. 1. Hydrophobic binding sites, patterned over a hydrophilic substrate submerged in an aqueous hosting liquid, are selectively coated with thin lenses of a hydrophobic fluid (in the following, the lubricant). Dies are delivered to the substrate and, upon stochastic contact of their hydrophobic sides with the lubricant lenses (1), capillarity (2) drives them to selfalign with the binding sites (3). Alignment accuracy better than $0.2 \mu \mathrm{m}$ was claimed at this stage of assembly [3].
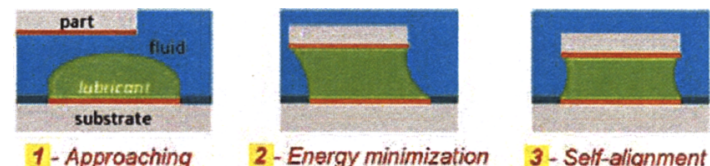

Fig. 1 Stages of capillary fluidic self-assembly.

Establishing reliable interconnects between dies and substrates is a central issue for the full development of capillary SA. Xiong et al [4] used a polymer for capillary alignment and mechanical binding of LEDs to the substrate, and post-assembly electroplating of solder vias for electrical interconnects, thus requiring extensive postprocessing and dedicated substrate structures. Fukushima et al. [5] enhanced pick-and-place with capillary selfalignment of dies for wafer reconstruction on a temporary carrier, followed by wafer-level alignment and transfer of dies to the target substrate, and substantial postprocessing to realize electrical connections. In moltensolder-driven SA [6] the high surface tension of a molten solder is used, normally in combination with die/substrate geometric shape-matching, to drive the assembly of components and establish, at the same time, electrical and mechanical interconnects. The assembly is performed at temperatures higher than the solder melting point. It thus suffers from continuous degradation of solder material (growth of oxides and intermetallics), which may be problematic for long assembly times and/or small-size interconnects.

We adopted a simple method for interconnects establishment in capillary SA originally proposed by Scott et al. [7]. It consists in embedding solder bumps inside the binding sites to be coated by the lubricant. After capillary assembly, substrate and assembled dies are transferred to a hot plate where a reflow-like treatment enforces solder bonding. The method decouples assembly and bonding: it performs the assembly at room temperature, and realizes electrical and mechanical interconnections at once.

In this paper, we extend the above mentioned method beyond the original setting, where lead-based solder bumps were formed inside the binding sites by dipcoating. We opted for indium, as we required a lead-free, $\mathrm{Au}$-wetting solder with good electromechanical properties [8] and relatively low melting point $\left(156^{\circ} \mathrm{C}\right)$. Also, we preferred masked electroplating over dip-coating of solder bumps because of better control of dimensions, easier integration in production lines and lower thermal budget during wafer processing. 
In the following sections, after the description of the fabrication and assembly procedures, we present mechanical characterization and structural analysis of interconnects as established by the capillary SA process. A simple test structure designed to assess electrical functionality of interconnects is then described, and further discussion is finally provided.

\section{Materials and methods}

For the fabrication of both dummy dies and substrates, we started with clean 8 ', $725 \mu$ m-thick silicon wafers with a $200 \mathrm{~nm}$-thick thermal silicon dioxide top layer. $\mathrm{Ti} / \mathrm{Au}$ layers (50/100 nm-thick) were evaporated on top.

As for substrates, the metal layer was used as seed for subsequent masked electroplating of bumps. As underbump metallurgy, we used a Ni diffusion barrier $(1 \mu \mathrm{m})$ and an Au adhesion layer (300 nm). An In layer, $7 \mu \mathrm{m}$ thick on average, was then electrodeposited. As-plated In roughness was as high as $0.5 \mu \mathrm{m}\left(\mathrm{R}_{\mathrm{a}}\right)$. Bumps diameters ranged from 50 to $400 \mu \mathrm{m}$. Several centro-symmetric bump arrays were designed. The seed layer was later lithographically patterned and etched to define a $1 \times 1 \mathrm{~mm}^{2}$ square binding site around each bump array, the sites being spaced by silicon dioxide areas. The wafers were then diced into chips containing arrays of sites and, after standard cleaning, immersed overnight in a $1 \mathrm{mM}$ ethanolic solution of dodecanethiol self-assembling monolayers (SAMs), which assemble preferentially over $\mathrm{Au}$ making it hydrophobic [9]. Water contact angles (CAs) measured on test substrates were $\sim 110^{\circ}$ for SAMtreated $\mathrm{Au}$ surfaces and $<10^{\circ}$ for silicon dioxide. A large difference in surface energy is a prerequisite for selective coating of chemically heterogeneous substrates [10].

As for dies, the starting wafers were backside thinned down to $200 \mu \mathrm{m}$, diced into $1 \times 1 \mathrm{~mm}^{2}$ square dies and treated as above to make their Au side hydrophobic. Native silicon oxide rendered the other sides hydrophilic. Being mainly interested in solder/metal wetting and, moreover, having to address a variety of solder bump arrays on the same substrate chips, in this study we did not pattern bonding pads on Au die sides. Both substrates and dies were stored in ethanol after preparation.

All our experiments were performed in uncontrolled laboratory atmosphere. In such conditions, In surface is covered by a few nanometer-thick, self-passivating native oxide, which prevents solder reflow and thus solder/metal wetting. Our solution to this problem consisted in a chemical removal of the oxides from the solder surface before selectively dip-coating the binding sites with a non-reducing fluid. We dipped the substrates in $10 \% \mathrm{wt}$. $\mathrm{HCl}(\mathrm{pH}<2)$ for 5 ' to etch the solder oxides, rinsed them with DI water and ethanol, dried them with nitrogen and immediately slid them through an air/hexadecane (HD)/water interface, thus forming stable lenses of HD (Aldrich, purity $>99 \%$ ) [10]. The substrates were left in water. Comparison between Figures $2 \mathrm{a}$ and $2 \mathrm{~b}$ shows that the presence of arbitrary arrays of solder bumps do not influence the dip-coating of binding sites. This holds true as long as the lenses are thicker than the bumps, and the bumps are placed away from the edges of the sites. Notably, SAMs layers were essentially unaltered after the exposure to diluted $\mathrm{HCl}$, which indeed only caused a reduction of the average measured water CAs to $108^{\circ}$.

Dies were subsequently extracted from ethanol and directed in water toward the substrates, where they wandered and assembled aided by fluidic stirring. Upon assembly, dies stand on top of the HD lenses and do not contact the bumps. Assembly events are stochastic, and site filling is asymptotic. High filling rates require an excess of dies as compared to sites. Unbound dies can be carefully removed by laminar fluid flow and eventually recycled for other assembly runs.

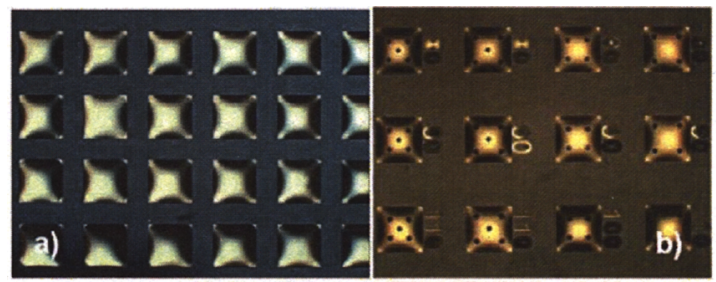

Fig. 2 HD lenses over binding sites in water (side: $1 \mathrm{~mm}$ )

\section{Post-assembly solder bonding}

After assembly, substrates look hydrophilic, since assembled dies show their hydrophilic sides to the surface and are separated by hydrophilic areas. Thus, upon extraction from a clean water/air interface, the substrates are covered by a water film. It preserves die alignment during substrate movement in air. Its thickness depends on the velocity and angle of extraction of the substrates with respect to the water surface. At this stage, the dies are still floating above the bumps.

Two bonding methods were tested.

In the first, proposed by Scott et al. [7], the substrates were simply set on a hot plate (HP) and reflowed. We kept the temperature of the hot plate at $95^{\circ} \mathrm{C}$ for 3 ' to let all residual water evaporate from dies and substrate. After almost complete water removal, HD spreads marginally over the spacer area around the sites. Upon subsequent, rapid increase of temperature, $\mathrm{HD}$ was completely removed at temperatures between $150^{\circ}$ and $160^{\circ} \mathrm{C}$, far below its boiling point $\left(287^{\circ} \mathrm{C}\right)$. Finally, the temperature was kept at $200^{\circ} \mathrm{C}$ for $3^{\prime}$ (as suggested in [8]) to enforce bonding. Results were checked after slow cooling of the samples. Fig. $3 \mathrm{a}$ and $3 \mathrm{~b}$ shows a substrate chip before and after HP treatment, respectively. It can be seen that die placement is overall preserved.

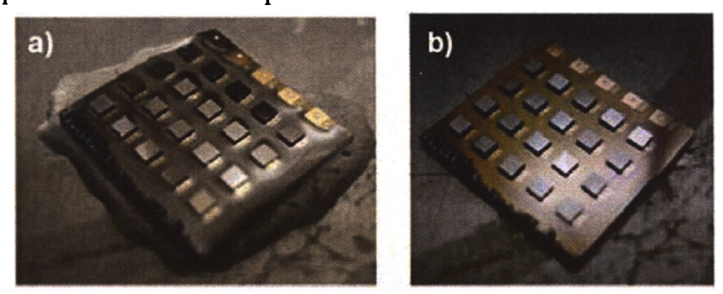

Fig. 3 Bonding on hot plate: (a) before and (b) after reflow 
As second method, we tested thermocompression (TC) bonding. The substrates were mounted on the chuck of a flip-chip bonder. While still at room temperature, the upper arm applied a force collectively on all dies. The force was defined so to apply a pressure of $2.5 \mathrm{MPa}$ on each bump. Bonding was then performed following the same thermal profile as before. Both chuck and arm were held at the same temperature during the treatment.

\section{Mechanical characterization of interconnects}

We did not reflow the bumps before processing since, being the bumps very thin, excessive formation of $\mathrm{In} / \mathrm{Au}$ intermetallics (IMCs), which form readily even at room temperatures [11], and their diffusion toward the surface could prevent In from melting and wetting Au.

After the full treatment explained above, nearly all assembled dies subjected to both bonding methods were stably attached to the substrate, independent of array configuration. Bonding yield was anyway apparently lower for sparse arrays with bumps of small diameters (< $100 \mu \mathrm{m}$ ), due to an easy disruption of the solder joints upon substrate manipulation. This observation suggests that, for such conditions, the use of proper die underfilling to avoid premature bond failures is mandatory.

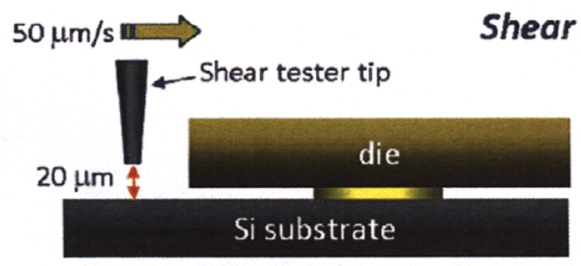

Fig. 4 Automated shear test set-up
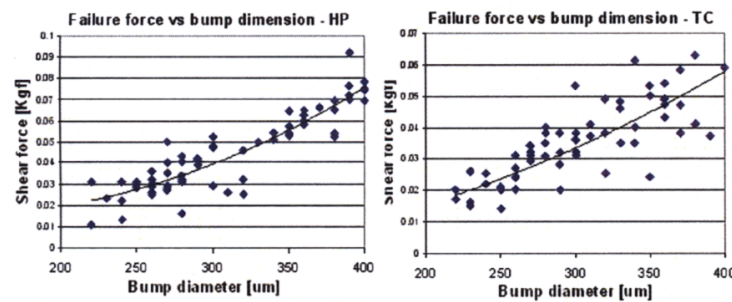

Fig. 5 Measured shear force vs bump diameter.

To check the mechanical performance, we subjected the bonded samples to an automated shear test. As shown in Fig. 4, the shear tester tip acted on the side of the die and was positioned $20 \mu \mathrm{m}$ above the substrate plane, so that the shear movement of die would be as parallel to the substrate plane as possible; the shear rate was $50 \mu \mathrm{m} / \mathrm{s}$. The tests were performed at room temperature on dies bonded over sites containing a single, central bump. We wanted to ascertain preliminarily whether the adhesion of the dies to the substrate would be due to fluid-mediated stiction. Shear test results are shown in Fig. 5 for both HP and TC bonding. The quadratic relation between maximum shear force and bump diameter supports the absence of stiction. Furthermore, no traces of residual HD were detected in any sheared samples. Thus, completely removing HD is straightforward, as compared to common fluxing agents. Shear trace data measured for bumps with diameters smaller than $200 \mu \mathrm{m}$ were very noisy and difficult to interpret. Thus they were discarded from statistics. Fig. 6 compares the shear strength distributions measured for both HP- and TC-bonded dies. Measured shear strength values were in line with literature on $\mathrm{In} / \mathrm{Au}$ bumps [8]. A summary of data is presented in Table 1 .

Table I Summary of shear test data for In bumps

\begin{tabular}{|c|c|c|}
\hline Bump diameter $[\mu \mathrm{m}]$ & \multicolumn{2}{|c|}{$220 \div 400$} \\
\hline Bump height $[\mu \mathrm{m}]$ & \multicolumn{2}{|c|}{$9 \pm 1$} \\
\hline Bonding method & Hot plate & TC \\
\hline \# of measured dies & 67 & 60 \\
\hline Avg. strength $[\mathrm{MPa}]$ & 5.58 & 4.73 \\
\hline Std. deviation $[\mathrm{MPa}]$ & 1.12 & 0.98 \\
\hline
\end{tabular}

Shear strength distribution

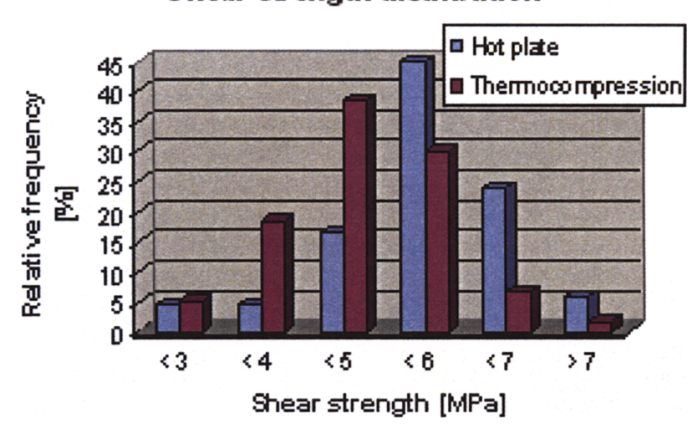

Fig. 6 Shear strength distributions of In bumps

\section{Structural characterization of interconnects}

To investigate the structure of the bumps after bonding, we analyzed dies cross-sections with SEM and EDX. Figures $7 \mathrm{a}$ and $7 \mathrm{~b}$ show examples of $9 \mu \mathrm{m}$-thick bumps bonded to die by HP and TC, respectively. In our experience, it was difficult to avoid embedding hard particles (mainly, silicon carbide) into the soft solder layer during sample preparation. FIB milling of the surface of the cross-sections partly removed the exposed In, leaving voids in its place. The overall appearance of the bumps is similar in the two cases. As clearly visible, the $\mathrm{Au}$ layer deposited on top of the $\mathrm{Ni}$ layer was consumed reacting with In and forming large grains of InAu IMCs, which, probably as consequence of ripening, then diffused across most of the bump height. EDX analysis showed that these IMCs are In-rich; this is coherent with the equilibrium phase diagram of the $\mathrm{In} / \mathrm{Au}$ system [11] and the composition of our bumps, which had excess In content compared to $\mathrm{Au}$. The grains are enclosed in a matrix of pure In. No evident trace of In/ $/ \mathrm{Ni}$ IMCs was detected at the $\mathrm{Ni}$ interface, probably due to limited reflow time as compared to the slow reaction time of Ni with In [12]. On the die side, the Au layer was also consumed. Interestingly, a layer of unreacted In was still present in the upper part of the bump. Thus, under the 
adopted reflow conditions $\mathrm{Au} / \mathrm{In}$ IMCs spread through the bumps, except for the top part where a thin layer of pure In is available for wetting and bonding. The large amount of IMCs, tougher than pure In, may explain the high shear strength values measured for the bumps [8].

Figures $8 \mathrm{a}$ and $8 \mathrm{c}$ show SEM imaging of the $\mathrm{Au}$ surface of dies sheared after HP and TC bonding, respectively. The solder bump profile is replicated on the die surface with fidelity. A wetting precursor layer [13] is also seen spreading along the edge of the solder imprint, testifying the good quality of the In/Au wetting achieved with both bonding procedures. Upon shearing, bulk In, from 1 to $4 \mu \mathrm{m}$ in thickness as measured by profilometer, is transferred to the die surface, as better seen in Fig. $8 \mathrm{~b}$.

Moreover, the stripes on the surface of transferred In are evidence of the ductile behaviour of In under shear. In most of examined cases, the plane of failure under shear was entirely inside bulk In. In fewer cases, as in Fig. 8c, the failure plane also intersected the bump/die interface, exposing a region of interfacial In/Au IMCs formed at the time of wetting. Again, the behaviour under shear for both HP and TC samples was similar.
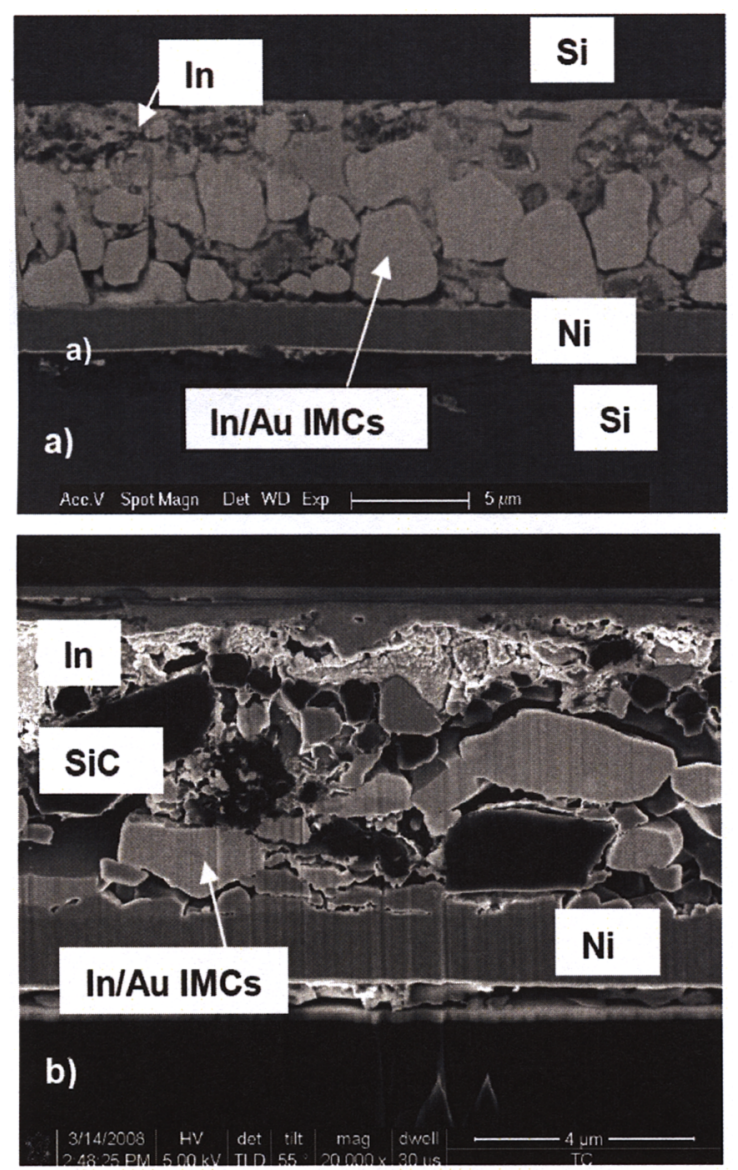

Fig. 7 Cross-section of In bumps: (a) HP and (b) TC bonding
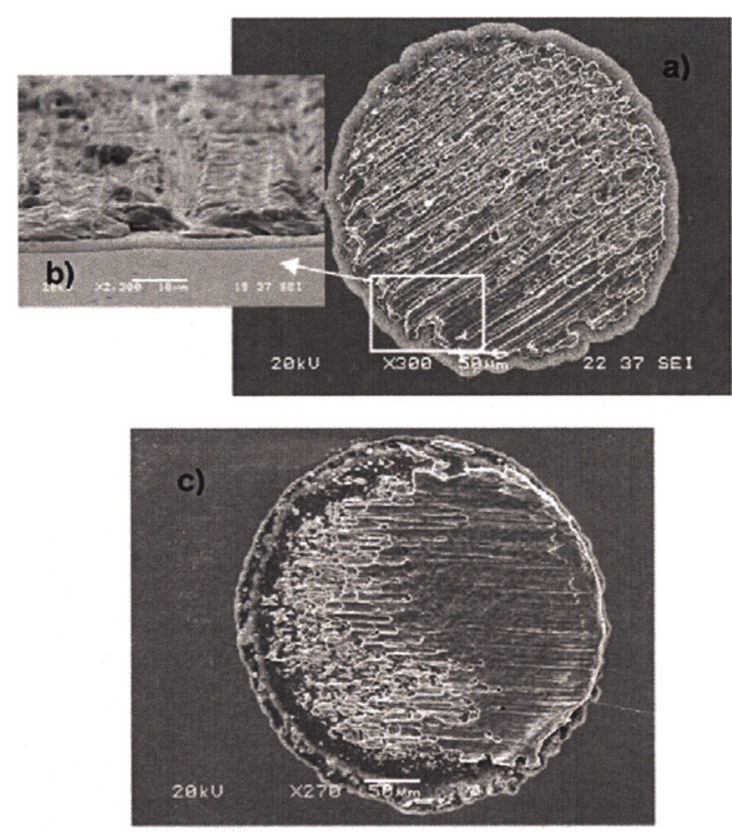

Fig. 8 Imprints of In bumps on sheared dies: (a) HP (b) TC

Finally, scanning acoustic microscopy revealed the occasional presence of small voids in the In bumps, just below the die/solder interface (data not shown). Voids may be a consequence of the use of as-plated, rough bumps and/or of incomplete solder reflow, eventually combined with liquid entrapment at some process stage. Voids seem more common in HP than in TC samples, possibly due to the higher applied load during TC bonding. Additional post-bonding reflow may be required to reduce voiding. Voids influence on shear performance was not characterized, though it may be significant.

\section{Electrical functionality of solder interconnects}

To verify the electrical functionality of In solder interconnects established in our process, we fabricated a simple daisy-chain-like test structure. The matching patterns of the $1 \times 1 \mathrm{~mm}^{2}$ binding sites and dies are shown in Fig. $9 a$ and $9 b$, respectively. They were etched on a $\mathrm{Ti} / \mathrm{Ni} / \mathrm{Au}$ layer $(50 / 500 / 50 \mathrm{~nm}$-thick; measured sheet resistance: $0.67 \Omega / \square$ ) over silicon oxide. The binding sites included 4 electroplated In bumps (diameter: $120 \mu \mathrm{m}$ ). Their position matched that of equal-sized bonding pads on the dies. The bumps were externally addressable by independent gold pads. Substrates and dies were treated as described in Section 2. An HD-coated binding site and the final, self-assembled and bonded structure are shown in Fig. 9c and 9d, respectively. Though this binding site design is not optimal for conformal dip-coating [14], we opted for it because of ease of processing. 

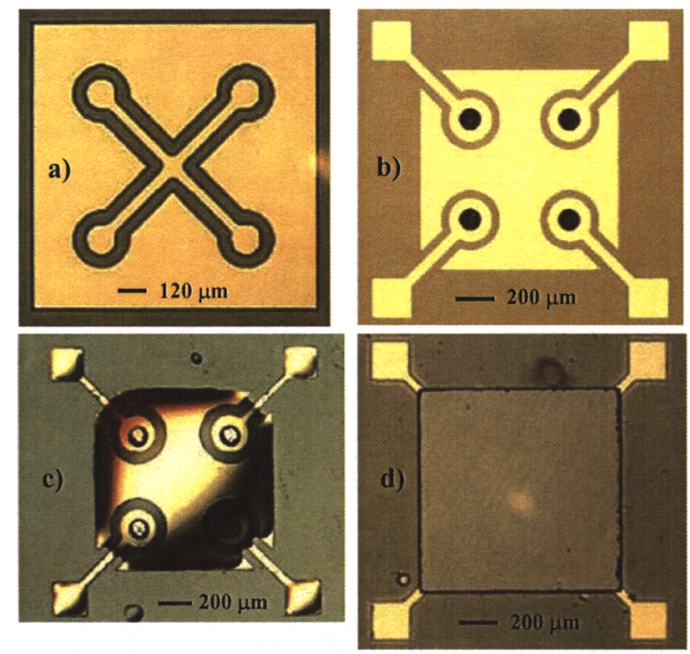

Fig. 9 Assessment of electrical interconnect. (a) Bonding pads patterned on die. (b) Corresponding binding site with 4 electroplated In bumps. (c) Binding site dip-coated with hexadecane (in water). (d) Self-assembled and bonded die.

Bonding was performed with both procedures described above. After correct assembly and alignment of dies, the electrical paths linking each pair of external pads would ideally be identical. On bonded samples, 2terminal measurements between pairs of external pads gave a resistance value of $28 \pm 2.8 \Omega$. According to a simplified model of the structure, where the entire resistance of the electrical paths is attributed to the thin metal layers, we expected a resistance value of $\sim 27$ $\Omega$ (Fig. 10). While the agreement is satisfying, the discrepancy with the measured values may be due to the contact resistance of the measurement tool and tolerances in the dimensions of the metal layer. While we saw no significant difference in measured resistance values between HP and TC samples, HP ones showed a higher deviation from the average.

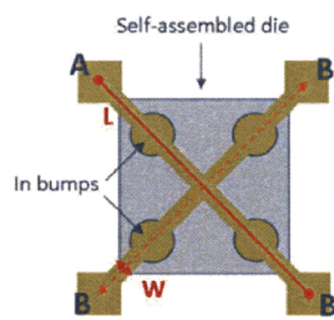

$$
\begin{array}{ll}
\mathrm{L}_{\mathrm{AB}}=1300 \sqrt{2} \mu \mathrm{m} & \mathbf{R}_{\mathrm{s}}: 0.67 \Omega / \square \\
\mathbf{W}=45 \mu \mathrm{m} & \mathbf{R}_{\mathrm{AB}}=\mathrm{R}_{\mathrm{s}} * \mathrm{~L} / \mathrm{W} \sim 27.3 \Omega
\end{array}
$$

Fig. 10 Simplified model of the test structure

Overall, though not being able to determine In bumps contact resistance, we could reasonably prove electrical functionality of interconnects.

\section{Discussion}

During HP reflow, no load is applied on the dies other than their own negligible weight and the temporary hydrostatic pressure provided by the residual fluids. Working in uncontrolled atmosphere, we found that successful bonding depended critically on the thermal profile of reflow, as it controlled the dynamics of both HD removal and solder reoxidation. We experimentally observed that the dies came into contact with bumps only after almost complete HD removal, i.e. at the beginning of solder reoxidation. As expected, bonding yield was highest when we could keep the HD on the samples up to temperatures as high as In melting point, to enforce metal wetting before consistent solder reoxidation. Notably, at this temperature the dodecanethiol SAM should have already desorbed from the Au surface [15]. Accordingly, under these conditions the wetting between molten In and Au surfaces should ideally take place in absence of any intervening layer (SAMs, HD or oxide). Conversely, in TC bonding the contact between solder and Au takes place already at room temperature. It is thus mediated by the alkanethiol monolayer. Interestingly, this appears not to be an obstacle to subsequent In/Au wetting, as showed in section 4 . We did not investigate the actual fate of the organic interlayer, though. The applied pressure should also squeeze out the HD from between the solder/die interface and enforce a more complete contact between the surfaces, so as to prevent solder reoxidation even with early HD removal.

We note that, though the boiling point of HD is $287^{\circ}$ $\mathrm{C}$, depending on their size the HD lenses completely evaporated at much lower temperatures - around $160^{\circ} \mathrm{C}$ in our experience. We do not expect significant differences with the use of longer chain hydrocarbons. Therefore, performing HP bonding in a reducing atmosphere or with very low oxygen concentration might be mandatory for using higher melting point solders, besides allowing for flexible reflow profiles. Preliminary experiments conducted using tin bumps (melting point: $232^{\circ} \mathrm{C}$ ) seem to support this observation. Indeed, after preparing the samples as described in Sections 2 and 3, $\mathrm{Sn} / \mathrm{Au}$ bonding between bumps and dies was achieved only for dies reflowed under forming gas atmosphere.

We observed that the type of bonding influenced the alignment of the dies on the binding sites. In TC bonding, the applied external load should ideally freeze the position of the dies putting them in contact with the bumps. In practice, imperfect parallelism between chuck and arm of the bonder may cause lateral displacement of dies, especially if the dies stand in a vertically-tilted position. In HP bonding, the final position of the dies is sensitive to the dynamics of removal of fluids. The 2stage reflow profile was purposely defined to gently let the water, kept on the substrate after assembly, evaporate avoiding die dislodgment due to its high surface tension. During water evaporation, dies may also collapse together into clusters, as consequence of the capillary action of the liquid menisci bridging them. Such immersion force [16] is dependent on the size of and the distance between the 
dies. Also, HD evaporation from between the dies and the substrate sites can be non-uniform and can be responsible for a slight tilt and misplacement in the position of the dies. Finally, die-to-substrate alignment is directly limited by dicing and lithographical tolerances $( \pm 5 \mu \mathrm{m}$ using the current method). This prevented us in the first place from estimating the achievable alignment accuracy.

\section{Conclusions}

In this paper we presented for the first time analysis and characterization of lead-free solder interconnects established as an integral part of a capillary die-tosubstrate self-assembly process. According to the experimental evidence shown, we conclude that: 1) the issue of the oxide passivating the solder bumps can be simply and effectively overcome by chemically removing the oxide before coating the bumps with a non-reducing fluid, which needs to be preserved during assembly and bonding; 2) alkanethiol SAMs seem not to prevent metallic wetting between oxide-free In and Au surfaces; 3) no substantial difference in mechanical and electrical performance exists between dies bonded with and without external application of additional load during bonding, though better reliability and reproducibility may be achieved in the former case. The last observation may open interesting perspectives for the application of the technique to the assembly of dies over e.g. curved or stretchable substrates, which cannot easily tolerate the imposition of substantial force for bonding purposes.

Future work will aim at further optimizing bonding parameters, investigating the use of alternative fluids to enhance assembly and reflow steps, and estimating the accuracy achievable in die alignment after solder bonding.

\section{References}

1. Savastiouk, S., Siniaguine, O., Korczynski, E, "3D stacked wafer-level packaging", Advanced packaging, March 2000, pp. 28-34.

2. Morris, C. Y., Stauth, S. A., Parviz, B. A., "SelfAssembly for Microscale and Nanoscale Packaging: Steps Toward Self-Packaging" IEEE Trans. Adv. Pack., Vol. 28, No. 4 (2005), pp. 600-611.

3. Srinivasan, U., Liepmann, D., Howe, R. T., "Microstructure to substrate self-assembly using capillary forces" IEEE Journal of MEMS, Vol. 10, No. 1 (2001), pp.17-24.

4. Xiong, X., Hanein, Y., Fang, J., Wang, Y., Wang, W., Schwartz, D. T., Bohringer, K. F., "Controlled multibatch self-assembly of microdevices", IEEE Journal of MEMS, Vol. 12, No. 2 (2003), pp.117-128.

5. Fukushima, T., Yamada, Y., Kikuchi, H., Tanaka, T., Koyanagi, M., "Self-Assembly Process for Chip-toWafer Three-Dimensional Integration", Proc. $57^{\text {th }}$ Eletronic Components and Technology Conf., Orlando, FL, May 2007, pp. 836-841.

6. Saeedi, E., Abbasi, S., Bohringer, K. F., Parviz, B. A., "Molten-Alloy Driven Self-Assembly for Nano and
Micro Scale System Integration", FDMP, Vol.2, No.4 (2006), pp.221-245.

7. Scott, K. L., Hirano, T., Yang, H., Singh, H., Howe, R. T., Niknejad, A. M., "High-performance inductors using capillary based fluidic self-assembly", IEEE Journal of MEMS, Vol. 13, No. 2 (2004), pp. 300-309

8. Liu, C.-C., Lin, Y.-K., Houng, M.-P., Wang, Y.-H., "The microstructure investigation of flip-chip laser diode bonding on silicon substrate by using Indiumgold solder", IEEE Trans. CPT, Vol. 26, No. 3 (2003), pp. 635-641.

9. Love, J. C., Estroff, L. A., Kriebel, J. K., Nuzzo, R. G., Whitesides, G. M., "Self-assembled monolayers of thiolates on metals as a form of nanotechnology", Chem. Rev., Vol. 105 (2005), pp. 1103-1169.

10. Biebuyck, H. A., Whitesides, G. M., "SelfOrganization of Organic Liquids on PatternedSelfAssembled Monolayers of Alkanethiolates on Gold", Langmuir, Vol. 10 (1994), pp. 2790-2793.

11. Okamoto, H., Massalski, T. B., Phase Diagrams and binary Gold alloys, ASM International, 1987, pp. 142153.

12. Wakiyama, S., Ozaki, H., Nabe, Y., Kume, T., Ezaki, T., Ogawa, T., "Novel low-temperature CoC interconnection technology for multichip LSI (MCL)", Proc. 57 $7^{\text {th }}$ Electronic Components and Technology Conf., Orlando, FL, May 2007, pp. 610615.

13. Liu, Y. M., Chuang, T. H., "Interfacial reactions between liquid indium and Au-deposited substrates", J. Electr. Mat., Vol. 29, No. 4 (2000), pp. 405-410.

14. Mastrangeli, M., Ruythooren, W., Baert, K., Van Hoof, C., Celis, J.-P., "Improving conformality in selective dip-coating of patterned planar substrates", Proc. Foundation of Nanoscience 2007, Snowbird, Utah, April 2007, pp. 230-237.

15. Delamarche, E., Michel, B., Kang, H., Gerber, C., "Thermal stability of self-assembled monolayers", Langmuir, Vol. 10 (1994), pp. 4103-4108.

16. Kralchevsky, P. A., Nagayama K., "Capillary forces between colloidal particles", Langmuir, Vol. 10 (1994), pp. 23-36. 